\title{
A formação do profissional fisioterapeuta na atenção à saúde do idoso: uma revisão integrativa
}

\author{
Magali Grave*, Cristianne Famer Rocha", Eduardo Périco***
}

\section{Resumo}

O objetivo desta pesquisa é analisar a produção científica brasileira sobre a atual formação do fisioterapeuta no que diz respeito à atenção à saúde do idoso. Este estudo consiste em uma revisão integrativa da literatura, no qual foram acessadas as bases de dados eletrônicas LILACS, SciELO e Scopus, utilizando-se os descritores "fisioterapia e saúde do idoso" (physiotherapy elder health, reabitacion física, salud del idoso), "educação superior" (education higher, educación superior), "educação em saúde" (health education, educación en salud) em artigos publicados entre 2000 e 2011. Foi selecionado um total de 60 artigos, sendo 20 deles excluídos. Dos 40 restantes, 30 respondiam parcialmente ao conteúdo proposto no estudo e 10 contemplavam integralmente a questão norteadora da pesquisa. Apesar de ser imperativa a qualificação na área da saúde do idoso, persiste a presença de pouco conteúdo de Geriatria e Gerontologia nos currículos dos cursos de graduação em Fisioterapia, assim como no dos demais da área da saúde. A maior responsabilidade fica com a extensão universitária e a pós-graduação lato sensu e stricto sensu. Nesse sentido, fazem-se necessárias a revisão e a reformulação dos projetos pedagógicos dos cursos de graduação, com vistas à qualificação de futuros profissionais.

Palavras-chave: Fisioterapia baseada em evidência. Saúde do idoso. Pesquisa. Educação superior. Educação em saúde.

\section{Introdução}

O Sistema Único de Saúde (SUS), implementado no Brasil pela Constituição Federal de 1988, tem como princípios a universalidade, a integralidade e a equidade e está organizado sob as diretrizes da descentralização, do atendimento integral e da participação da comunidade. (MENICUCCI, 2009). A criação do SUS desencadeou uma completa reorganização do sistema para a atenção à saúde da população nas políticas públicas, no processo de trabalho e na formação dos profissionais envolvidos na saúde. Em decorrência, ao longo das

* Fisioterapeuta. Mestra em Desenvolvimento Regional - UNISC - RS. Doutoranda em Ciências da Saúde - PUCRS. Docente vinculada ao Centro de Ciências Biológicas e da Saúde do Centro Universitário Univates - Lajeado - RS. Endereço para correspondência: Rua Avelino Tallini, 171, Bairro Universitário, CEP: 95.900-000, Lajeado - RS. E-mail: mgrave@univates.br.

** Enfermeira. Doutora em Educação - UFRGS. Professora adjunta da UFRGS, vinculada ao curso de Análise de Políticas e Sistemas de Saúde/bacharelado em Saúde Coletiva.

*** Biólogo. Mestre em Genética - UFRGS. Doutor em Ecologia USP - SP. Coordenador do Programa de Pós-Graduação - mestrado/doutorado em Ambiente e Desenvolvimento - Univates - Lajeado - RS.

$\hookrightarrow$ doi:10.5335/rbceh.2012.039 
últimas décadas, inúmeras transformações políticas, sociais, econômicas e demográficas vêm ocorrendo no Brasil, tendo estas repercussão nas condições de vida e de trabalho da população e, consequentemente, na sua situação de saúde. (CAMPOS, 2003).

Ao mesmo tempo, a formação dos profissionais da área da saúde tem sido alvo importante para o êxito nas transformações propostas pelo SUS. A formação profissional, ainda orientada por uma concepção pedagógica que estabelece o centro das aprendizagens no hospital universitário, hierarquiza os adoecimentos em critérios biologicistas e dissocia clínica e política. (CECCIM; FERLA, 2009).

Com relação ao profissional fisioterapeuta - por sua própria concepção e conformação ao modelo assistencial curativo -, a fisioterapia destinou-se quase exclusivamente ao controle dos danos de determinadas doenças. A atuação na fisioterapia reabilitadora é destinada à cura de doenças e/ou à reabilitação de sequelas e complicações. Seu objeto de intervenção é, na maioria das vezes, o sujeito individualizado, quando não apenas partes ou órgãos isolados do seu corpo. (BISPO JÚNIOR, 2010).

Rever o processo de formação desse profissional é extremamente importante em qualquer situação de saúde da população. Todavia, considerando que o envelhecimento populacional é um fenômeno mundial, que as tendências demográficas demonstram uma crescente linear nos índices de envelhecimento entre os anos de 1980/2000 e que o profissional fisioterapeuta poderá atuar tanto na prevenção, quanto na promoção e reabilitação dessa população, faz-se necessário priorizar ações que contemplem a saúde do idoso. A tendência é que o Brasil, em 2025, seja o sexto país do mundo em número de idosos. (MARIN; CECÍLIO, 2009). A Lei n. 10.741, de $1^{\circ}$ de outubro de 2003 , que dispõe sobre o Estatuto do Idoso, Capítulo V, artigo 22, recomenda que, nos currículos dos diversos níveis de ensino formal, sejam inseridos conteúdos voltados ao processo de envelhecimento, ao respeito e à valorização do idoso, regulamentando a capacitação de profissionais no campo da Gerontologia. Além disso, no seu capítulo IV, artigo 15, assegura a atenção integral à saúde do idoso por intermédio do SUS, garantindo-lhe 0 acesso universal igualitário em conjunto articulado e contínuo das ações e dos serviços para a prevenção, promoção, proteção e recuperação da saúde, incluindo a atenção especial às doenças que afetam preferencialmente os idosos. (BRASIL, 1999).

A partir da recente conjuntura de mudanças e transformações por que passa o campo das políticas públicas, da formação de profissionais e das práticas de saúde, com o paulatino avanço da consolidação do SUS, o profissional fisioterapeuta tem ampliado seu campo de atuação para além da reabilitação com enfoque na prevenção de doenças e promoção de saúde. Na condição de integrante de uma equipe multiprofissional, o fisioterapeuta poderá colaborar tanto com a equipe de saúde como no cuidado ao idoso, atuando em programas de educação em saúde e oportunizando a melhora da capacidade funcional, da segurança e da qualidade de vida dessa população. Dessa forma, a atuação da fisioterapia na terceira idade represen- 
ta uma estratégia importante para um estilo de vida mais saudável e ativo, com vistas a um envelhecer com saúde, maior qualidade e independência funcional. (CONDRADE; APRILE, 2010). Sendo assim, busca-se aqui, por meio de uma revisão integrativa, investigar como está ocorrendo a formação dos profissionais na área da saúde - em especial do fisioterapeuta -, para que se alcance a qualificação da atenção ao idoso. A questão norteadora deste estudo é: "o que as pesquisas científicas abordam sobre a relação da formação profissional em fisioterapia com a qualificação na atenção à saúde do idoso?".

\section{Metodologia}

Este é um estudo do tipo revisão integrativa da literatura, cuja metodologia caracteriza-se pela busca do pesquisador em sumarizar os resultados de uma pesquisa sobre o mesmo tema, a partir da síntese e análise dos dados encontrados nos estudos já publicados. (COOPER, 1982).

As bases de dados eletrônicas utilizadas para a busca dos artigos científicos foram a Literatura Latino-Americana e do Caribe em Ciência e Saúde (LILACS), a Scientific Eletronic Library Online (SciELO) e a base de dados Scopus, devido à sua confiabilidade, à atualização dos periódicos indexados e ao fato de conterem publicações nacionais e internacionais na área da saúde em português, inglês e espanhol.

Os descritores foram definidos com base nos Descritores em Ciências da Saúde (DeCS) e no Centro Latino-Americano e do Caribe de Informação em
Ciências da Saúde da (Bireme) e incluíram os termos: "fisioterapia e saúde do idoso" (physiotherapy elder health, reabitacion física salud del idoso), "educação superior" (education higher, educación superior) e "educação em saúde" (health education, educación en salud).

Os critérios de inclusão foram: artigos na área da saúde publicados em periódicos nacionais e internacionais abordando a temática da formação de profissionais fisioterapeutas na saúde do idoso no período de 2000 a 2011, resultantes de pesquisas primárias e secundárias; relatos de experiência; estudos teóricos; artigos na íntegra com acesso livre on-line em texto completo e sem custos para o acesso; artigos que responderam à questão norteadora deste estudo e que, caso necessário, tenham sido submetidos e aprovados por um Comitê de Ética em Pesquisa.

No total, foram selecionados 60 artigos, dos quais 20 foram excluídos por não serem de livre acesso on-line, por não apresentarem texto completo e/ou por estarem fora do período proposto (entre 2000 e 2011). Dos 40 artigos restantes, 30 respondiam parcialmente ao proposto no estudo e 10 contemplavam integralmente a questão norteadora da pesquisa.

O registro das informações baseou-se em um instrumento estruturado, padronizado e validado por Ursi (2005), contendo itens que identificam pontos relevantes do artigo, tais como: título, periódico, ano de publicação, idioma, instituição sede do estudo, características metodológicas, tipo de publicação, objetivo, amostra, dentre outros. Esse instrumento foi individualmente preenchido pela pesquisadora após a leitu- 
ra do conteúdo dos artigos na íntegra, tendo como objetivo a formulação do problema do estudo. Cada artigo analisado e incluído no estudo recebeu uma numeração sequencial.

A pesquisadora elaborou um quadro sinóptico no qual os campos destinam-se ao registro do resumo das informações contidas no instrumento utilizado para a coleta de dados, com as seguintes variáveis: numeração, título do artigo, autor, ano de publicação, metodologia, principais resultados e limitações. Essa análise caracterizou-se pela síntese, comparação e discussão das informações obtidas dos artigos incluídos na amostra deste estudo. Da análise preliminar, surgiram três eixos temáticos: 1) mudanças na formação a partir da implementação do SUS; 2) atenção à saúde do idoso por profissionais da área da saúde; 3 ) formação profissional do fisioterapeuta em relação à saúde do idoso.

\section{Resultados e discussão}

\begin{tabular}{|c|c|c|c|}
\hline Autor(es) & Título do artigo & Periódico & Ano \\
\hline $\begin{array}{l}\text { Coury, H. J. C. G.; } \\
\text { Vilela, I. }\end{array}$ & $\begin{array}{l}\text { Perfil do pesquisador fisioterapeuta } \\
\text { brasileiro }\end{array}$ & $\begin{array}{l}\text { Revista Brasileira de } \\
\text { Fisioterapia }\end{array}$ & 2009 \\
\hline $\begin{array}{l}\text { Aveiro, M. C.; } \\
\text { Acioli, G. G.; } \\
\text { Driusso, P. }\end{array}$ & $\begin{array}{l}\text { Perspectivas da participação do } \\
\text { fisioterapeuta no Programa Saúde da } \\
\text { Família na atenção à saúde do idoso }\end{array}$ & Ciência \& Saúde Coletiva & 2011 \\
\hline Trelha, C. S. et al. & $\begin{array}{l}\text { O processo de trabalho do } \\
\text { profissional fisioterapeuta }\end{array}$ & Revista Espaço para Saúde & 2002 \\
\hline Chaimowicz, $\mathrm{F}$. & $\begin{array}{l}\text { Fisioterapia e saúde coletiva: } \\
\text { desafios e novas responsabilidades } \\
\text { na formação profissional }\end{array}$ & Ciência \& Saúde Coletiva & 2010 \\
\hline $\begin{array}{l}\text { Silva, D. J.; } \\
\text { Da Ros, M. A. }\end{array}$ & $\begin{array}{l}\text { A inserção de profissionais } \\
\text { fisioterapeutas na Equipe de Saúde } \\
\text { da Família e Sistema Único de } \\
\text { Saúde: desafios na formação }\end{array}$ & Ciência \& Saúde Coletiva & 2008 \\
\hline Ribeiro, K. S. Q. & $\begin{array}{l}\text { A atuação da fisioterapia na } \\
\text { atenção ao idoso }\end{array}$ & Fisioterapia Brasil & 2002 \\
\hline $\begin{array}{l}\text { Cachioni, M.; } \\
\text { Neri, A. L. }\end{array}$ & $\begin{array}{l}\text { Educação e Gerontologia: desafios } \\
\text { e oportunidades }\end{array}$ & $\begin{array}{l}\text { Revista Brasileira do } \\
\text { Envelhecimento Humano }\end{array}$ & 2004 \\
\hline $\begin{array}{l}\text { Menezes, R. L.; } \\
\text { Souza, M. R.; } \\
\text { Rodrigues, T.; } \\
\text { Cardoso, C. }\end{array}$ & $\begin{array}{l}\text { O conhecimento de acadêmicos de } \\
\text { fisioterapia em relação à velhice e } \\
\text { ao envelhecimento. }\end{array}$ & $\begin{array}{l}\text { Revista Fragmentos de } \\
\text { Cultura }\end{array}$ & 2007 \\
\hline $\begin{array}{l}\text { Blascovi, A.; } \\
\text { Peixoto. }\end{array}$ & $\begin{array}{l}\text { A visão dos pacientes no atendimento } \\
\text { de fisioterapia: dados para traçar um } \\
\text { novo perfil profissional }\end{array}$ & $\begin{array}{l}\text { Revista Fisioterapia em } \\
\text { Movimento }\end{array}$ & 2002 \\
\hline Bispo Júnior, J. P. & $\begin{array}{l}\text { Fisioterapia e saúde coletiva: } \\
\text { desafios e novas responsabilidades } \\
\text { profissionais }\end{array}$ & Ciência \& Saúde Coletiva & 2010 \\
\hline
\end{tabular}

Quadro 1 - Apresentação e caracterização dos artigos selecionados conforme autor(s), título, periódico, ano e delineamento da pesquisa. 
Como é possível visualizar no Quadro 1 , os dez artigos que contemplavam integralmente o propósito deste estudo foram publicados nos periódicos Revista Brasileira de Fisioterapia, Revista Brasileira Ciência do Envelhecimento Humano, Ciência \& Saúde Coletiva, Espaço para Saúde, Fisioterapia em Movimento, Fisioterapia Brasil e Revista Fragmentos de Cultura, num período que variou de 2002 a 2011.

Mudanças na formação a partir da implementação do SUS

Com a implantação do SUS, torna-se necessário que mudanças ocorram na formação dos profissionais da área da saúde. Porém, para Menicucci (2009), essas mudanças têm permanecido alheias à organização da gestão setorial e ao debate crítico sobre os sistemas de estruturação do cuidado. As instituições formadoras têm perpetuado modelos essencialmente conservadores, centrados em aparelhos e sistemas orgânicos e tecnologias altamente especializadas, dependentes de procedimentos e equipamentos de apoio diagnóstico e terapêutico.

$\mathrm{O}$ autor acima citado refere que, para que essa mudança ocorra efetivamente, a formação desses profissionais não pode tomar como referência apenas a busca eficiente de evidências ao diagnóstico, cuidado, tratamento, prognóstico, etiologia e profilaxia das doenças e dos agravos. Deve, cada vez mais, buscar desenvolver condições de atendimento às necessidades de saúde das pessoas e das populações, da gestão setorial e do controle social em saúde, redimensionando o desenvolvimento da autonomia das pessoas até a condição de influência na formulação de políticas do cuidado.

Além disso, para Salmória e Camargo (2008) há de se considerar que o conceito de saúde, nas últimas décadas, vem passando por intensas transformações - principalmente no que diz respeito ao modelo de saúde até então adotado -, saindo de um modelo hospitalocêntrico, curativo e reabilitador para transformar-se em um modelo assistencial promotor da saúde, preventivo e, principalmente, contando com a participação popular e a interdisciplinaridade dos diferentes profissionais da saúde. Nesse contexto, entende-se saúde não como o avesso da doença, mas como a busca do equilíbrio do ser humano - devendo, portanto romper os estreitos limites da assistência curativa.

No Brasil, o setor da saúde vem sendo submetido a um significativo processo de reforma de Estado, protagonizado por importantes segmentos sociais e políticos, cuja ação é fundamental à continuidade e ao avanço do movimento pela Reforma Sanitária e concretização do SUS. Por essa razão, as várias instâncias desse sistema devem cumprir um papel indutor no sentido da mudança, tanto no campo das práticas de saúde como no da formação profissional. (CECCIN, 2009).

No setor do ensino, entretanto, revela-se necessário e urgente semelhante movimento social por uma Reforma da Educação que expresse o atendimento dos interesses públicos no cumprimento das responsabilidades de formação acadêmico-científica, ética e humanística, deixando clara a demanda para o desempenho tecnoprofissional. (CAM- 
POS, 2003). Faz-se premente, então, a reformulação dos Projetos Político-Pedagógicos dos cursos da área da saúde. A crescente procura por profissionais capazes de atuar com qualidade, resolutividade e em trabalho multiprofissional e interdisciplinar desencadeou a criação das novas diretrizes curriculares para os cursos na área da saúde, apontando para essa nova realidade e necessidade social. (CECCIN, 2009; CAMPOS, 2003).

Atenção à saúde do idoso por profissionais da área da saúde

Há de considerar, na nova realidade social comentada anteriormente, o novo perfil demográfico e epidemiológico do país - que indica um aumento da população idosa que, em síntese, advém das transformações ocorridas no século XX. Dessa forma, ocorreram o aumento na urbanização, o decréscimo na fecundidade e na mortalidade infantil e alterações nos padrões de saúde e doença, refletindo no aumento da expectativa de vida. (TAVARES, 2008).

Conforme dados da Organização Mundial de Saúde (OMS), o Brasil terá 32 milhões de pessoas idosas em 2025 - fato que impõe a necessidade de atendimento qualificado a essa população, evidenciada por meio da Política Nacional do Idoso (PNI), da Lei de Diretrizes Básicas da Educação e da própria demanda nos serviços de saúde. (BRASIL, 1999). Por isso, as questões ligadas à velhice e ao processo de envelhecimento, atualmente, representam um desafio para estudiosos e pesquisadores de diferentes áreas de atuação profissional. Em relação à área da saúde, destaca-se, na
PNI, a disposição que contempla a capacitação de recursos humanos para atuar nas unidades geriátricas de referência, ressaltando-se que esses profissionais necessitam ter formação especializada nas áreas de Geriatria e Gerontologia Social, a fim de assistir adequadamente essa clientela. (FREITAS; MAUYAMA, 2002).

A promoção de saúde e a profilaxia primária e secundária de doenças, inclusive após os 65 anos, são as alternativas que apresentam o melhor custo-benefício para que se alcance a compressão da morbidade. Sua importância deve ser enfatizada nos cursos de graduação e de educação continuada da área da saúde, sendo necessário que os profissionais estejam devidamente preparados para prestar cuidados ao idoso - já que essa faixa etária apresenta uma instalação muito rápida dos processos patológicos. (CHAMOVICS, 2000).

Para o autor, os profissionais da saúde, geralmente, não visualizam o idoso como um indivíduo que apresenta necessidades diferentes dos demais adultos; consequentemente, os estudantes não são estimulados a aplicar conhecimento e conceitos específicos relacionados à Gerontologia em sua dinâmica assistencial. Torna-se necessário, então, desenvolver atividades acadêmicas que não apenas informem acerca do envelhecimento, mas que também formem profissionais capazes de respeitar os limites e as peculiaridades decorrentes do envelhecimento, tornando-os aptos a reconhecer as modificações físicas, emocionais e sociais do idoso. 
Considerando essa necessidade, a PNI propõe modificações curriculares que têm como objetivos produzir conhecimento e discutir o processo de envelhecimento, eliminando preconceitos. (RAMOS; PERRACINI; KALACHE, 2000). Assim, torna-se necessário voltar a atenção da academia para a formação e a capacitação de recursos humanos, com vistas ao atendimento do fenômeno do envelhecimento (Gerontologia) e ao processo saúde-doença dos idosos (Geriatria). Pesquisas têm evidenciado a necessidade da capacitação de recursos humanos para prestar assistência a essa população - tanto no âmbito profissional como no leigo - junto às comunidades e às famílias. Portanto, as instituições de ensino superior, os centros formadores de opiniões e de profissionais devem promover, continuamente, discussões acadêmico-científicas direcionadas à investigação das questões associadas ao processo de envelhecimento, à velhice $\mathrm{e}$ de políticas acadêmicas voltadas para a formação de profissionais envolvidos com o segmento idoso. (RAMOS; PERRACINI; KALACHE, 2000).

Segundo Ribeiro (2002), nos cursos de graduação da área da saúde, ainda persiste, de maneira geral, a presença de pouco conteúdo de Geriatria e Gerontologia nos currículos, assim como há escassez de campos específicos para a prática. Um estudo realizado com instituições de ensino público de cursos de graduação da área da saúde do estado de Minas Gerais verificou que essa temática tem sido abordada em $75 \%$ deles, em disciplinas específicas. As investigações e a legislação brasileira, a exemplo da PNI e do Estatuto do Idoso, têm evidenciado a necessidade de adequar a formação de recursos humanos em saúde, de forma a sistematizar as competências profissionais para a atenção à saúde dos idosos. (TAVARES et al., 2008).

Formação profissional do fisioterapeuta em relação à atenção à saúde do idoso

No intuito de buscar alternativas e novas propostas de formação e atuação profissional para o alcance do propósito da PNI, foram definidas diretrizes essenciais à promoção do envelhecimento saudável. Foram essas a manutenção da capacidade funcional, a assistência às necessidades de saúde do idoso, a reabilitação da capacidade funcional comprometida, a capacitação de recursos humanos especializados, o apoio ao desenvolvimento de cuidados informais e o apoio a estudos e pesquisas. (COURY; VILELA, 2009).

Dentre os profissionais responsáveis pelo enfrentamento na diminuição de agravos - com vistas à melhora da qualidade de vida, prevenção de complicações e facilitação da independência funcional dessa população - está o fisioterapeuta. A sua formação foi, durante muito tempo, voltada para a prática médica com uma abordagem biológica e intra-hospitalar, potencializada pela sua própria gênese, evolução histórica, legislação e pelo seu currículo nos cursos de graduação. Recentemente, em face das transformações por que passa o campo da saúde, com a organização do SUS, a fisioterapia tem atuado para além da reabilitação. (AVEIRO; ACIOLI; DRIUSSO, 2011; TRELHA, 2002). 
Nos últimos anos, a fisioterapia vem ampliando e aprofundando seus conhecimentos técnicos e alargando sua área de atuação. No entanto, essa ampliação ocorreu, majoritariamente, no nível terciário. Mesmo com a expansão das possibilidades de atuação do profissional, ainda predomina uma atenção destinada à recuperação de distúrbios ortopédicos, traumatológicos e neurológicos. Porém, diante dos novos desafios da sociedade brasileira, surge a necessidade do redimensionamento do objeto de intervenção da fisioterapia - que deve se aproximar do campo da promoção da saúde e da nova lógica de organização dos modelos assistenciais, sem, porém, abandonar suas competências concernentes à reabilitação. (CHAMOVICS, 2000).

Essa preocupação também é encontrada no debate da formação acadêmica dos fisioterapeutas, como destacado por Salmória e Camargo (2008). Há certo consenso em relação à ampliação do objeto de trabalho do fisioterapeuta na formação. Segundo Silva e Da Ros (2007), as Diretrizes Curriculares Nacionais para os Cursos de Graduação em Fisioterapia, aprovadas em 2002, asseguram uma formação generalista. Os conteúdos curriculares podem ser diversificados, desde que assegurem $o$ equilíbrio de conhecimento em diferentes áreas, níveis de atuação e recursos terapêuticos. (AVEIRO; ACIOLI; DRIUSSO, 2011; TRELHA, 2002; CHAMOVICS, 2000; SALMÓRIA; CAMARGO, 2008).

Ribeiro (2002) chama a atenção para o fato de que o conteúdo curricular nos cursos de graduação em Fisioterapia vol- tados à atenção ao idoso deveria assumir caráter relevante, visto que constitui ferramenta substancial à compreensão dos fenômenos que envolvem essa população. Porém, ao contrário do que se pretende, a maioria dos cursos de graduação em Fisioterapia conta com, no máximo, uma ou duas disciplinas relacionadas ao idoso - e nestas, o conteúdo está centrado na reabilitação de disfunções cardiorrespiratórias, musculoesqueléticas e prevenção de quedas. (NERI; JORGE, 2006).

Há de se considerar que as possibilidades de atuação do fisioterapeuta caminham para sua adequação à política pública de saúde preconizada pelo SUS, na qual a valorização da prevenção e a promoção de saúde - assim como a busca da equidade e maior resolutividade dos atendimentos prestados - representam os principais elementos norteadores das ações propostas. (CACHIONI; NERI, 2004).

Em recente estudo sobre a inserção de acadêmicos de Fisioterapia em serviços de saúde direcionados à população idosa, foi possível identificar que aqueles alunos que não haviam sido previamente preparados com tal conteúdo apresentavam dificuldades em lidar com determinadas situações. Diante da realidade demográfica e epidemiológica do país, é inquestionável a necessidade do estudo da Geriatria e da Gerontologia em cursos de graduação. No Brasil, a Universidade é um dos principais agentes sociais empenhados em propor programas direcionados à população idosa, principalmente em projetos de extensão. $\mathrm{Na}$ área em pauta, não tem sido diferente: muitas 
das ações de assistência em Fisioterapia à população idosa estão concentradas em postos de saúde, Estratégias de Saúde da Família (ESF), casas de longa permanência e clínicas-escola por meio da realização de estágios curriculares obrigatórios e não obrigatórios e projetos de extensão. (SALMÓRIA; CAMARGO, 2008).

Poucos docentes de Fisioterapia estão envolvidos em cursos de pós-graduação estudando Fisioterapia Gerontológica, e a maioria que ministra tal conteúdo o faz com base em sua experiência, em autoestudo e educação continuada. (NERI; JORGE, 2006). Semelhantemente a esse fato, observou-se, no estudo de Ribeiro (2002), que a maioria dos acadêmicos de Biomedicina (76,8\%), de Enfermagem $(70,9 \%)$, de Medicina (66\%) e de Fisioterapia $(70,2 \%)$ da Universidade Federal da Bahia não sabia a diferença entre Geriatria e Gerontologia.

As Diretrizes Curriculares do Curso de Fisioterapia contemplam o domínio de conhecimentos de fisiologia, procedimentos diagnósticos físico-funcionais e terapêuticos necessários à prevenção, promoção e reabilitação das doenças de maior prevalência epidemiológica e aspectos da saúde do idoso. Porém, para Neri e Jorge (2006), a formação do profissional de saúde que atua na atenção ao idoso deve ter como base o perfil do gerontólogo, por ele apontado como aquele apto a: aprender, histórica e criticamente, o processo do envelhecimento em seu conjunto; compreender o significado social da ação gerontológica; situar o desenvolvimento da Gerontologia no contexto sócio-histórico; atuar nas expressões da questão da velhice e do envelhecimento, formulando e implementando propostas para o enfrentamento; realizar pesquisas que subsidiem a formulação de ações gerontológicas; compreender a natureza interdisciplinar da Gerontologia, buscando ações compatíveis no ensino, na pesquisa e na assistência, dentre outras. Esse perfil é baseado nos objetivos da educação gerontológica, os quais podem ser sintetizados na intenção de formar recursos humanos capazes de compreender o "ser velho" e o processo de envelhecimento em suas dimensões conceituais, sociais, políticas, profissionais e éticas.

O currículo dos cursos de saúde, relacionado às formas de organização do envelhecimento, é um "artefato social e cultural". Não deve ser entendido como transcendente e atemporal - ele tem uma história vinculada a formas específicas e contingentes de organização da sociedade e da educação e, por isso, deve ser flexível e repensado em diferentes momentos históricos. Esse é um desafio para coordenadores, docentes e discentes do Ensino Superior, planejadores de programas de educação permanente nos diversos serviços, sobretudo quando se considera a atual escassez, nos currículos de formação superior em saúde, de conteúdos e cenários de prática relacionados à Geriatria e à Gerontologia. (MENEZES; SOUZA, CARDOSO, 2007; BLASCOVI; PEIXOTO, 2002; BISPO JÚNIOR, 2010; SANTOS; BULB; MENDES, 2000; CACHIONI; JORGE, 2006). 


\section{Conclusão}

É de fundamental importância que as Instituições de Ensino Superior (IES) revisem e repensem seus projetos pedagógicos, bem como os conteúdos desenvolvidos sobre a temática do idoso. $\mathrm{O}$ momento é oportuno para a busca de opções inovadoras nos campos da Geriatria e da Gerontologia, dadas as mudanças demográficas e epidemiológicas pelas quais o Brasil está passando. Refletir sobre a formação acadêmica geriátrica e gerontológica é perceber o ensino como instância que procura transmitir uma cultura que permita ao futuro profissional respeitar a condição humana e pensar de forma contextualizada, aberta, globalizada e ética. Nesse sentido, a fisioterapia geriátrica e gerontológica deve ter como meta a reinserção social em aspectos preventivos, reabilitadores e curativos, mas, para romper o ciclo da doença, é necessária uma abordagem que ultrapasse o modelo biomédico e valorize os aspectos sociais e multidimensionais do indivíduo idoso.

Nos cursos de graduação da área da saúde, de maneira geral, ainda persiste a presença de pouco conteúdo de Geriatria e Gerontologia nos currículos, assim como há escassez de campos específicos para a prática, representando a carência de recursos humanos um grave problema na assistência ao idoso. A complexidade e a extensão dos cuidados a essas pessoas exigem conhecimentos sobre o envelhecimento e as formas de adaptação desses indivíduos a tal processo. Escassos conhecimentos nas áreas da Geriatria e da Gerontologia darão origem a ava- liações equivocadas sobre a velhice e refletir-se-ão em preconceito em relação ao envelhecimento - o que, por sua vez, resultará em formas de tratamento, práticas e políticas inapropriadas a essa população.

As especificidades do processo de envelhecimento humano necessitam do atendimento nas múltiplas dimensões, abarcando, entre outros aspectos, os sociais, políticos, culturais e econômicos. Nessa perspectiva, abrem-se, além de novos espaços de atuação para o profissional de saúde, novas abordagens metodológicas - tais como a interdisciplinaridade, que busca superar a fragmentação do conhecimento, na medida em que favorece a troca de saberes entre as diversas áreas profissionais no entendimento de uma dada realidade.

The physical therapist professional training in elderly health care: an integrative review

\section{Abstract}

The objective of this study was to analyze the Brazilian scientific production on the current lineup of the physiotherapist in the health care of the elderly. An integrative literature review was done, in which the electronic databases LILACS, SciELO and Scopus were accessed using the descriptors, physical therapy and health of the elderly (physiotherapy elder health, reabitacion física, salud del idoso), higher education (educação superior), health education (educação em saúde, educacion em salud) in papers published between 2000 and 2011. A total of 60 papers were selected, 20 being excluded. Of the remaining 40 articles, 30 responded only partially to the proposed in the study and 10 fully contemplated the question guiding the re- 
search. There is still low content of geriatrics and gerontology in the curricula of undergraduate courses in physiotherapy, as well as other health care, getting more responsibility with the university extension and post-graduation lato sensu and stricto sensu. In this sense, it is necessary to review and reformulation of pedagogical projects of undergraduate courses, aimed at the qualification of future professionals.

Keywords: Physical therapy. Elderly health care. Higher education. Health education

\section{Referências}

AVEIRO, M. C.; ACIOLI, G. G.; DRIUSSO, P. Perspectivas da participação do fisioterapeuta no Programa Saúde da Família na atenção à saúde do idoso. Ciência \& Saúde Coletiva, Rio de Janeiro, v. 16, Supl. 1, p. 1467-1478, 2011.

BISPO JÚNIOR, J. P. Fisioterapia e saúde coletiva: desafios e novas responsabilidades profissionais, Ciência \& Saúde Coletiva, Rio de Janeiro, v. 15, Supl. 1, p. 1627-1636, 2010.

BLASCOVI, A. S. M.; PEIXOTO, B. O. A visão dos pacientes no atendimento de fisioterapia: dados para traçar um novo perfil profissional. Fisioterapia em Movimento, Curitiba, v. 15, n. 1, p. 61-67, abr./set. 2002.

BRASIL. Ministério da Saúde. Portaria do Gabinete do Ministro de Estado da Saúde de $\mathrm{n}^{\circ} 1.395$, de 9 de dezembro de 1999. Aprova a Política Nacional de Saúde do Idoso e dá outras providências. Brasília: Diário Oficial [da] República Federativa do Brasil, Brasília, no 237-E, sessão 1, p. 20-24, 13 dez. 1999.

CACHIONI, M.; NERI, A. L. Educação e gerontologia: desafios e oportunidades. Revista Brasileira de Ciências do Envelhecimento Humano, Passo Fundo, v. 12, n. 4, p. 99-115, jan./jun. 2004.
CACHIONI, M.; JORGE, M. D. Atitudes e conhecimentos em relação à velhice em estudantes de graduação em educação e em saúde: subsídios ao planejamento curricular. Estudos de Psicologia, Campinas, v. 23, n. 2, p. 127-137, abr./jun. 2006.

CAMPOS, G. W. S. Paidéia e modelo de atenção: um ensaio sobre a reformulação do modo de produzir saúde. Olho Mágico, São Paulo, v. 10 , n. 2 , p. 7-14, abr./jun. 2003.

CECCIM, R. B.; FERLA, A. A. Educação e saúde: ensino e cidadania como travessia de fronteiras. Revista Trabalho, Educação e Saúde, Rio de Janeiro, v. 6, n. 3, p. 443-456, nov. 2008/fev. 2009.

CHAMOVICS, F. A saúde dos idosos brasileiros às vésperas do século XXI: problemas, projeções e alternativas. Revista de Saúde Pública, Porto Alegre, v. 84, n. 2, p. 184-200, 2000.

CONDRADE, T. V. L.; APRILE, M. R. Humanização da saúde na formação de profissionais da fisioterapia. Revista Equilíbrio Corporal e Saúde, São Paulo, v. 2, n. 2, p. 25-35, 2010.

COOPER, M. H. Scientific guidelines for conducting integrative research resiews. Review of Educational Research, Califórnia, v. 52, n. 2, p. 291-302,1982.

COURY, H. J. C. G.; VILELA, I. Perfil do pesquisador fisioterapeuta brasileiro. Rev Bras Fisioter, São Carlos/SP, v. 13, n. 4, p. 356-63, 2009.

FREITAS, M. C.; MAUYAMA, S. A. T. Perspectivas em gerontologia e geriatria: revisão de literatura. Revista Latino-Americana de Enfermagem, Ribeirão Preto, v. 10, n. 2, mar./ abr. 2002.

MARIN, M. J. S.; CECÍLIO, L. C. O. Necessidades de saúde de idosos de uma Unidade de Saúde da Família. Revista Brasileira de Geriatria e Gerontologia, Rio de Janeiro, v. 12 , n. 1, p. 12-18, 2009. 
MENEZES, R. L.; SOUZA, M. R.; CARDOSO, T. R. O conhecimento dos acadêmicos de fisioterapia em relação à velhice e ao envelhecimento. Fragmentos de Cultura, Goiânia, v. 17, n. 3/4, p. 293-301, mar./abr. 2007.

MENICUCCI, T. M. G. O Sistema Único de Saúde, 20 anos: balanço e perspectivas. Caderno de Saúde Pública, Rio de Janeiro, v. 25, n. 7, p. 1620-1625, jul. 2009.

NERI, A. L.; JORGE, M. D. Atitudes e conhecimentos em relação à velhice em estudantes de graduação em educação e em saúde: subsídios ao planejamento curricular. Estudos de Psicologia, Campinas, v. 23, n. 2, p. 127-137, abr./jun. 2006.

RAMOS, L. R.; PERRACINI, M. R. T. E.; KALACHE, A. Significance and management of disability among urban elderly residents in Brazil. Journal of Cross-Cultural Gerontology, Dordrecht, v. 8, n. 9, p. 313-323, 2000.

RIBEIRO, K. S. A atuação da fisioterapia na atenção primária à saúde. Fisioterapia Brasil, São Paulo, v. 3, n. 5, p. 311-318, 2002.

SALMÓRIA, J. G.; CAMARGO, W. A. Uma aproximação dos signos - Fisioterapia e Saúde - aos aspectos humanos e sociais. Saúde e Sociedade, São Paulo, v. 17, n. 1, p. 73-84, 2008.

SANTOS, L. L. C.; BULB, L. I. R. N.; MENDES, N. T. C. Levantamento dos conteúdos de Geriatria e Gerontologia dos currículos dos cursos de graduação em enfermagem em relação ao idoso apresentada por seus professores e estudantes. Revista Ciências da Saúde, São José do Rio Preto, v. 9, n. 2, p. 75-108, 2000.

SILVA, D. J.; DA ROS, M. A. A inserção de profissionais de fisioterapia na Equipe de Saúde da Família e Sistema Único de Saúde: desafios na formação. Ciência e Saúde Coletiva, Rio de Janeiro, v. 12, n. 6, p. 16731681, 2007.
TAVARES, D. M. S. et al. Ensino de gerontologia e geriatria: uma necessidade para os acadêmicos da área de saúde da Universidade Federal do Triângulo Mineiro? Revista Ciência Cuidado e Saúde, v. 7, n. 4, p. 537 545, out/dez 2008.

TRELHA, C. S. O processo de trabalho do profissional fisioterapeuta. Revista Espaço para Saúde, Londrina, v. 4, n. 1, p. 15-21, dez. 2002.

URSI, E. S. Prevenção de lesões de pele no perioperatório: revisão integrativa da literatura. 2005. 128f. Dissertação (Mestrado) - Universidade de São Paulo, Escola de Enfermagem de Ribeirão Preto, Ribeirão Preto, 2005. 